\title{
Combined treatment with tamoxifen and a fusicoccin derivative (ISIR-042) to overcome resistance to therapy and to enhance the antitumor activity of 5-fluorouracil and gemcitabine in pancreatic cancer cells
}

\author{
TAKAAKI MIYAKE ${ }^{1}$, YOSHIO HONMA ${ }^{1}$, TAKESHI URANO ${ }^{2}$, NOBUO KATO ${ }^{3}$ and JUNJI SUZUMIYA ${ }^{1}$ \\ Departments of ${ }^{1}$ Oncology/Hematology, ${ }^{2}$ Biochemistry, School of Medicine, Shimane University, Izumo, \\ Shimane 693-8501; ${ }^{3}$ The Institute of Scientific and Industrial Research, Osaka University, Osaka 567-0047, Japan
}

Received February 25, 2015; Accepted March 31, 2015

DOI: $10.3892 /$ ijo.2015.2979

\begin{abstract}
Although progress has been made in chemotherapeutic strategies against pancreatic cancer, overall survival has not significantly improved over the past decade. Thus, the development of better therapeutic regimens remains a high priority. Pancreatic cancer cell lines were treated with tamoxifen, a novel antitumor fusicoccin derivative (ISIR-042), and anticancer drugs, and their effects on cell growth, signaling and gene expression were determined. Xenografts of Panc-1 cells were treated with tamoxifen, ISIR-042 and 5-fluorouracil (5FU) to determine the effects on tumor growth. The inhibition of the growth of pancreatic cancer cells induced by tamoxifen was effectively reduced by $\alpha$-tocopherol, a membrane stabilizer. ISIR-042 produced synergistic effects with tamoxifen in inhibiting cell growth. Tamoxifen elevated lipid peroxidation and the release of cytochrome $\mathrm{c}$, and these effects of tamoxifen were reduced by $\alpha$-tocopherol. ISIR-042 significantly inhibited colony formation and the expression of stemness-related genes of pancreatic cancer cells. The triple combination of tamoxifen, ISIR-042, and 5FU or gemcitabine was effective at inhibiting cell growth and the appearance of drug-resistant cells. This combined treatment significantly inhibited the growth of Panc-1 cells as xenografts without apparent adverse effects. The triple combination of tamoxifen and ISIR-042 with 5FU or gemcitabine may be highly effective against pancreatic cancer by overcoming resistance to therapy.
\end{abstract}

\section{Introduction}

Pancreatic cancer remains a highly lethal neoplasm, and even with multimodality therapy for localized disease, patient

Correspondence to: Professor Yoshio Honma, Department of Oncology/Hematology, School of Medicine, Shimane University, 89-1 Enya, Izumo, Shimane 693-8501, Japan

E-mail: biohonma@med.shimane-u.ac.jp

Key words: tamoxifen, fusicoccin derivative, lipid peroxidation, pancreatic cancer cells, xenograft survival is measured in months. 5-Fluorouracil (5FU) and gemcitabine are the mainstays of therapy. However, the response rates to either are $<30 \%$, and their toxic effects are significant $(1,2)$. Although the FOLFIRINOX regimen has produced substantial benefits in the treatment of metastatic pancreatic cancer, it is associated with more adverse effects (3). Chemoresistance usually develops in patients who respond initially, and an effective salvage therapy is currently unavailable. Thus, the development of better therapeutic regimens for pancreatic cancer remains a high priority.

At the start of therapy, large pancreatic cancers always contain mixed populations of malignant cells that are resistant to anticancer agents, and these cells expand clonally once therapy is initiated. Tumor recurrence is thus a fait accompli when single agents are used. Combination therapy eliminates cells that are resistant to either drug alone, if there are no single mutations that cause cross-resistance to both drugs. Therefore, combination therapy with two drugs greatly increases the chance of success, since the likelihood of a doubly mutated cell emerging in such a population is low. However, mathematical analyses have revealed that triple therapy may be needed in patients with a large tumor burden (4).

Cotylenins, fusicoccins and some diterpene glucosides are modulators of 14-3-3 proteins, and have been shown to exhibit antitumor effects in vitro and in vivo. We previously synthesized many fusicoccin derivatives and found that ISIR-042 was the most potent at inhibiting the proliferation of tumor cells (5). ISIR-042 in combination with 5FU or gemcitabine was observed to have synergistic effects at inhibiting the growth of pancreatic cancer cells. In pancreatic cancers, late diagnosis and the early development of metastasis to the regional lymph nodes and liver limit curative resection to $<10 \%$ of patients. Since most of the patients have a large tumor burden, combination therapy with two drugs may be insufficient in these patients $(1,2)$. While the FOLFILINOX regimen has been introduced, this regimen is more toxic and few patients can receive it. Triple combination therapy with less toxicity may be required for an effective therapy against pancreatic cancers. Therefore, we examined the synergistic effects of various compounds and ISIR-042 on the growth of pancreatic cancer cells to identify the most potent and clinically applicable drugs. The 
most effective agent was tamoxifen. Tamoxifen is a selective estrogen receptor modulator that is used as the first-line treatment for estrogen receptor-positive breast cancer. However, multiple non-estrogen receptor-mediated mechanisms have been implicated in antitumor effects induced by tamoxifen in estrogen receptor-negative tumors (6). There seems to be a consistent relationship between higher doses of tamoxifen and longer survival in patients with largely inoperable and recurrent malignant glioma (7). Therefore, in the present study, we sought to clarify the synergistic effects of ISIR-042 and tamoxifen on human pancreatic cancer cells and to examine the therapeutic effects on xenografts of human pancreatic carcinoma cells in the presence or absence of 5FU treatment.

\section{Materials and methods}

Materials. ISIR-042 was prepared as previously described (5). Gemcitabine,tamoxifen,5FU, $\alpha$-tocopherol and 3-(4,5-dimethyl2-thiazolyl)-2,5-diphenyltetrazolium bromide (MTT) were purchased from Sigma-Aldrich Japan (Tokyo, Japan). Taurine, L-nitroarginine methyl ester (L-NAME), N-acetyl cysteine (NAC), and protease inhibitor cocktail were obtained from Wako Pure Chemical (Tokyo, Japan).

Cells and culture. Human pancreatic cancer cell lines CFPAC-1, BxPC-3 and Capan-2 were purchased from American Tissue Culture Collection (Manassas, VA, USA). Cells were cultured in RPMI-1640 medium (Sigma-Aldrich Japan) supplemented with $10 \%$ fetal bovine serum and $80 \mu \mathrm{g} / \mathrm{ml}$ gentamycin at $37^{\circ} \mathrm{C}$ in a humidified atmosphere of $5 \% \mathrm{CO}_{2}$ in air.

Colony-forming assay. MIAPaCa- 2 cells $\left(1 \times 10^{4}\right.$ per dish) were plated into $1.1 \mathrm{ml}$ of a semisolid methylcellulose medium with $0.8 \%$ methylcellulose and $20 \%$ fetal bovine serum in triplicate for 14 days. A solution of $0.2 \mathrm{ml}$ of PBS containing various concentrations of drugs was added to the semisolid medium. Colonies were photographed under an inverted microscope. Colonies in enlarged photographs were measured and counted. Colonies $>0.4 \mathrm{~mm}$ in diameter were counted.

Bone marrow plugs from a single femur of three Balb/c mice were pooled in RPMI-1640 medium. A single cell suspension was prepared by vigorous pipetting. Nucleated cells $(1,000 \mathrm{cells} / \mathrm{ml} / \mathrm{dish})$ were placed into a semi-solid medium containing hematopoietic growth factors (MethoCult ${ }^{\circledR} \mathrm{GF}$ M3434, Stem Cell Technology Inc., Vancouver, BC, Canada) and incubated for 10 days.

Assay of cell growth. Human cancer cells were seeded into 24 -well multidishes at $1.0 \times 10^{4}$ cells $/ \mathrm{ml} /$ well. The cells were cultured with various concentrations of drugs for 4-6 days. After culture with or without drugs, viable cells were examined by the MTT assay (5).

Analysis of the effects of combinations of drugs. An isobologram analysis was used to determine the effects of combinations of drugs on Panc-1 cells. Dose-dependent effects were determined for each compound and for one compound with fixed concentrations of another. The interaction of two compounds was quantified by determining the combination index (CI), in accordance with the following classic isobologram (8).
Assay of the cumulative cell number. The cell density of the drug-treated cells was kept at $2-8 \times 10^{4} / \mathrm{ml}$ to maintain the growth phase in a 24-well multidish (Falcon). The medium of treated cultures was replaced by fresh medium with drugs at least every 4 or 5 days to remove cell debris of dead cells. The viable cell number was measured by MTT assay. The cumulative cell number was calculated from the MTT values and the dilution used when feeding the culture.

Western blotting. Cells were packed after being washed with cold PBS and then lysed at $1.5 \times 10^{7}$ cells $/ \mathrm{ml}$ in sample buffer. The resultant lysate was resolved on SDS-polyacrylamide gel, as described elsewhere (5). Primary antibodies were obtained from the following sources: K-ras (Abnova, Taiwan); acid ceramidase (BD Biosciences Japan, Tokyo, Japan); and cytochrome c and actin (Cell Signaling Technology Japan, Tokyo, Japan).

Mitochondria preparation. After cells were washed twice with PBS, protease inhibitor cocktail was added and cells were homogenized by 30 strokes with a glass homogenizer. The homogenate was centrifuged at $1,000 \mathrm{x} \mathrm{g}$ and the pellet was discarded. The supernatant was centrifuged at $10,000 \mathrm{x} \mathrm{g}$ for $30 \mathrm{~min}$ and the cytoplasmic (supernatant) and mitochondrial (pellet) fractions were separated.

Cytochrome c release and lipid peroxidation. Cytochrome $\mathrm{c}$ was determined by western blot analysis using monoclonal anti-cytochrome c antibody. Lipid peroxidation (LPO) was determined by measuring thiobarbituric acid-reactive substances (TBARS) at $535 \mathrm{~nm}$, as described elsewhere (9).

Reverse transcription-polymerase chain reaction (RT-PCR). Total RNA was extracted from cells using TRI reagent (Sigma). Total RNA was converted to first-strand cDNA primed with random hexamer in a reaction volume of $20 \mu 1$ using an RNA PCR kit (qPCR RT Master Mix, Toyobo Co. Ltd., Osaka, Japan), and $2 \mu \mathrm{l}$ of this reaction was used as a template in realtime PCR. The primers used were previously described (10).

Transplantation of human cancer cells into nude mice. Fourweek-old female athymic nude mice with a BALB/c genetic background were obtained from CLEA Japan (Tokyo). They were housed under specific pathogen-free conditions. Mice were subcutaneously inoculated with $2 \times 10^{6}$ Panc- 1 cells. The adjusted $2 \times 10^{7}$ cells $/ \mathrm{ml}$ were mixed with an equal volume of Matrigel (BD Sciences) and $0.2 \mathrm{ml}$ of the cell solution was injected subcutaneously (11). Mice were given daily intraperitoneal injections of $3 \mathrm{mg} / \mathrm{kg}$ ISIR-042 in $0.2 \mathrm{ml}$ PBS and/or $2 \mathrm{mg} / \mathrm{kg}$ tamoxifen in $0.1 \mathrm{ml}$ corn oil, with the first treatment given 2 days after the inoculation of tumor cells (11). Treatment with $5 \mathrm{FU}$ was initiated when tumors in each group achieved an average volume of $80 \mathrm{~mm}^{3}$. Treatment with $5 \mathrm{mg} / \mathrm{kg} 5 \mathrm{FU}$ was performed three times per week. Tumor volume was measured with vernier calipers. Our protocol was approved by the animal ethics committee at Shimane University.

Statistical analysis. The results are expressed as means \pm standard deviation (SD). Pairs of data were compared using Student's t-test. Significant differences were considered to exist for probabilities $<5 \%(\mathrm{P}<0.05)$. For the in vivo experiment, an 
A
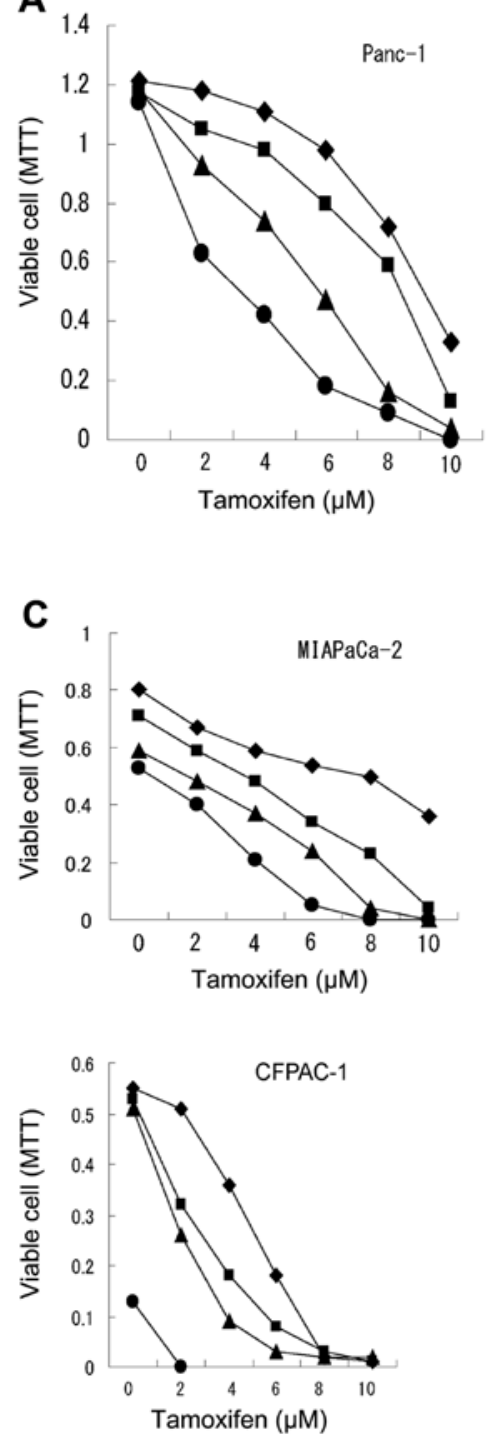

B
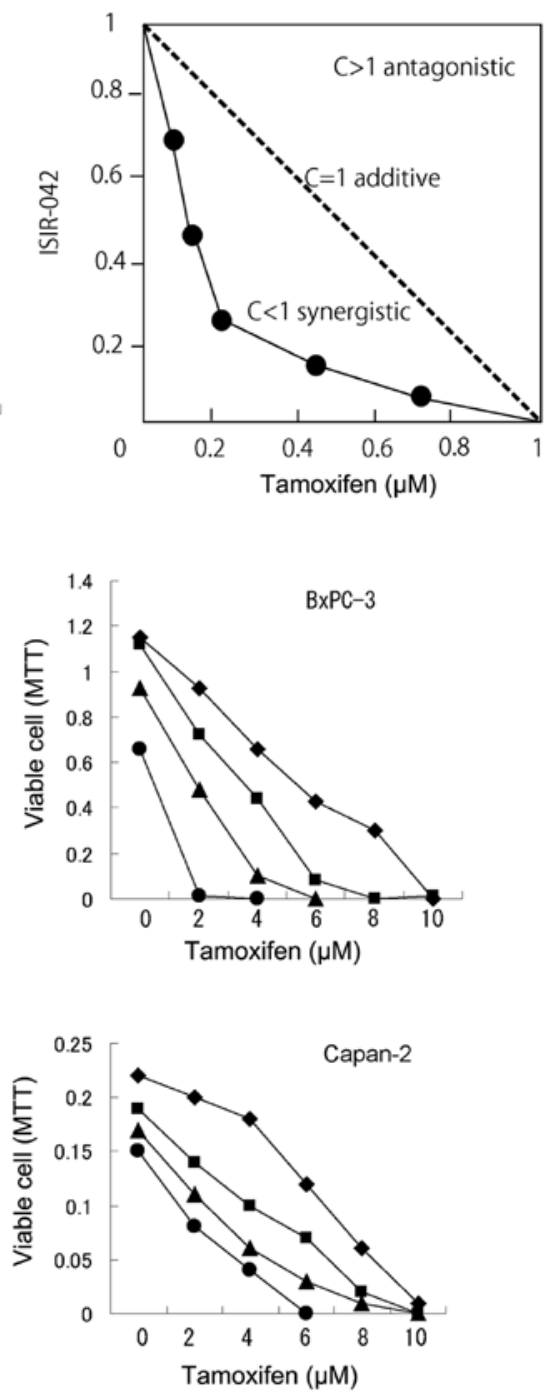

Figure 1. Combined effects of tamoxifen and ISIR-042 on the growth of pancreatic cancer cells. (A) Panc-1 cells were treated with various concentrations of tamoxifen in the presence of $0(\bullet), 2(\bullet), 4(\bullet)$, or $6(\bullet) \mu \mathrm{g} / \mathrm{ml}$ of ISIR-042 for 5 days. (B) Isobologram at IC I0 $_{50}$ Tamoxifen plus ISIR-42 in Panc-1 cells. Isoboles for the combination of tamoxifen with ISIR-042 that were isoeffective $\left(\mathrm{IC}_{50}\right)$ for inhibiting the proliferation of Panc-1 cells. The dashed line indicates zero interaction of the isobole. (C) Combined effects on other human pancreatic cancer cells. Cells were treated with various concentrations of tamoxifen in the presence of $0(\bullet), 2(\bullet), 4(\boldsymbol{\bullet})$, or $6(\bullet) \mu \mathrm{g} / \mathrm{ml}$ of ISIR-042 for 5 days.

F-test was performed to demonstrate statistical significance. Again, significant differences were considered to exist for probabilities $<5 \%$.

\section{Results}

Combined effects of tamoxifen and ISIR-042 on the growth of pancreatic cancer cells. Tamoxifen inhibited the growth of Panc-1 cells in a concentration-dependent manner, and the combined effects of tamoxifen and ISIR-042 are shown in Fig. 1. ISIR-042 produced synergistic effects with tamoxifen (Fig. 1A) and the results were confirmed by isobologram analysis. Fig. 1B shows isoboles for the combination of tamoxifen with ISIR-042 that were isoeffective $\left(\mathrm{IC}_{50}\right.$ : the concentration of the drug required for $50 \%$ inhibition of cell growth) for inhibition of the proliferation of Panc- 1 cells. These isoboles indicate that the combination of these drugs had synergistic effects. ISIR-042 and tamoxifen also had synergistic effects in other pancreatic cancer cell lines, although the sensitivity of pancreatic cancer cell lines to tamoxifen varied among the cell lines (Fig. 1C). Similar results were obtained when lung carcinoma and leukemia cells were treated with ISIR-042 and tamoxifen.

Tamoxifen enhances LPO and $\alpha$-tocopherol prevents the action of tamoxifen. Multiple non-estrogen receptor-mediated mechanisms have been implicated in cell death induced by tamoxifen (6). These mechanisms include changes in intracellular calcium, modulation of protein kinase $\mathrm{C}$, changes in calmodulin activity, signaling though mitogen-activated protein kinases including ERK, K-ras degradation (12) and inhibition of acid ceramidase activity (13). To understand the growth-inhibitory effect of tamoxifen on pancreatic cancer cells, we examined the effects of various compounds including estrogen and inhibitors of signaling on the growth of MIAPaCa- 2 cells in the presence of tamoxifen and/or ISIR-042. Estrogen did not affect the growth-inhibitory effect 
A

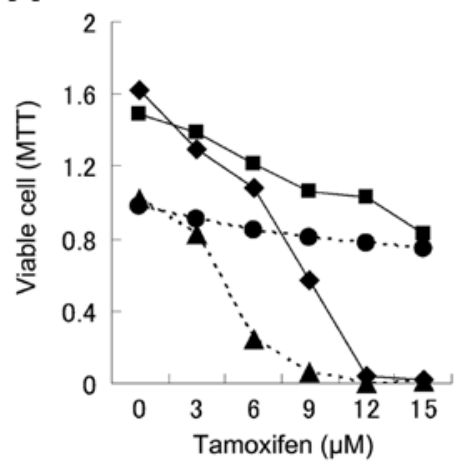

C
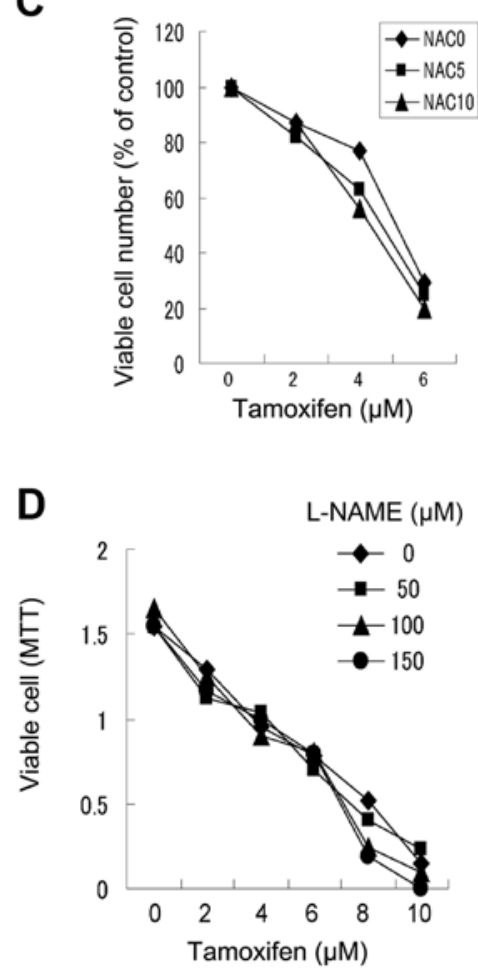

B
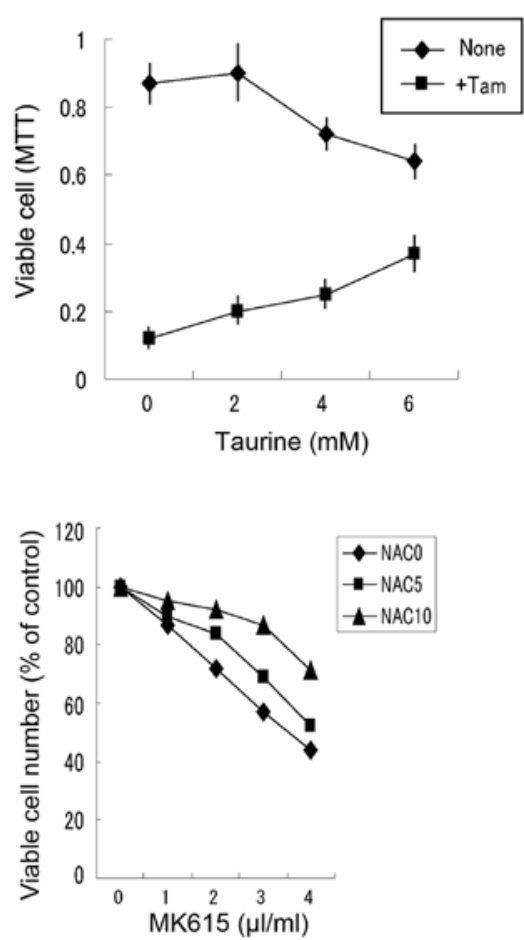

E

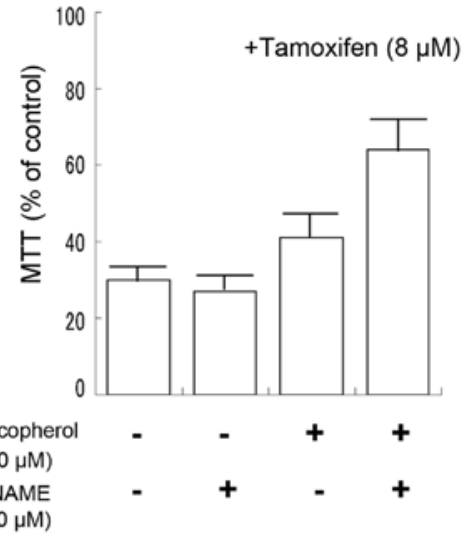

Figure 2. $\alpha$-tocopherol prevents tamoxifen-induced growth-inhibition. (A) MIAPaCa-2 cells were cultured with various concentrations of tamoxifen in the

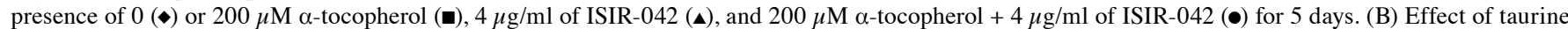
on tamoxifen-induced growth-inhibition in MIAPaCa-2 cells. Cells were cultured with ( $\mathbf{\square})$ or without $(\bullet) 9 \mu \mathrm{M}$ tamoxifen for 4 days. The values are means of four separate experiments \pm SD. (C) Effect of NAC on tamoxifen (left panel)- or MK615 (right panel)-induced growth-inhibition. MIAPaCa-2 cells were treated for 4 days. (D) Effect of L-NAME on tamoxifen-induced growth-inhibition in MIAPaCa-2 cells. (E) L-NAME enhances the effect of $\alpha$-tocopherol on tamoxifen-induced growth-inhibition in MIAPaCa-2 cells. Cells were treated for 4 days. The values are means of four separate experiments \pm SD.

of tamoxifen and/or ISIR-042, suggesting a non-estrogen receptor-mediated mechanism.

Several inhibitors of protein kinases also had no effect, whereas $\alpha$-tocopherol effectively reduced tamoxifen-induced growth inhibition but not ISIR-042-induced growth inhibition (Fig. 2A). Taurine, another membrane stabilizer and antioxidant, also reduced the growth inhibition induced by tamoxifen, although this effect was less than that of $\alpha$-tocopherol (Fig. 2B). When MIAPaCa- 2 cells were incubated with tamoxifen in the presence of the antioxidant NAC, tamoxifen-induced growth inhibition was not affected by NAC (Fig. 2C). However, treatment with NAC effectively inhibited the growth inhibition induced by the Japanese apricot extract MK615 (11) (Fig. 2C). Although the inhibitor of nitric oxide synthase L-NAME alone did not significantly affect tamoxifen-induced growth inhibition (Fig. 2D), LNAME effectively reduced tamoxifeninduced growth inhibition in the presence of a suboptimal concentration of $\alpha$-tocopherol (Fig. 2E). These results suggest that tamoxifen-induced changes in membrane dynamics are associated with growth inhibition in pancreatic cancer cells.

A recent study indicated that tamoxifen induced autophagic K-Ras degradation in many cancer cells including pancreatic cancer cells (12). Since pancreatic cancers exhibit an extremely high mutation rate of K-Ras (>90\%) (14), we examined the effect of $\alpha$-tocopherol on the degradation of K-Ras protein induced by tamoxifen in the presence or absence of ISIR-042. Tamoxifen concentration-dependently induced K-Ras degradation, and $\alpha$-tocopherol effectively counteracted this tamoxifen-induced degradation (Fig. 3A). ISIR-042 did not essentially affect the effects of $\alpha$-tocopherol and tamoxifen. These results suggest 
A
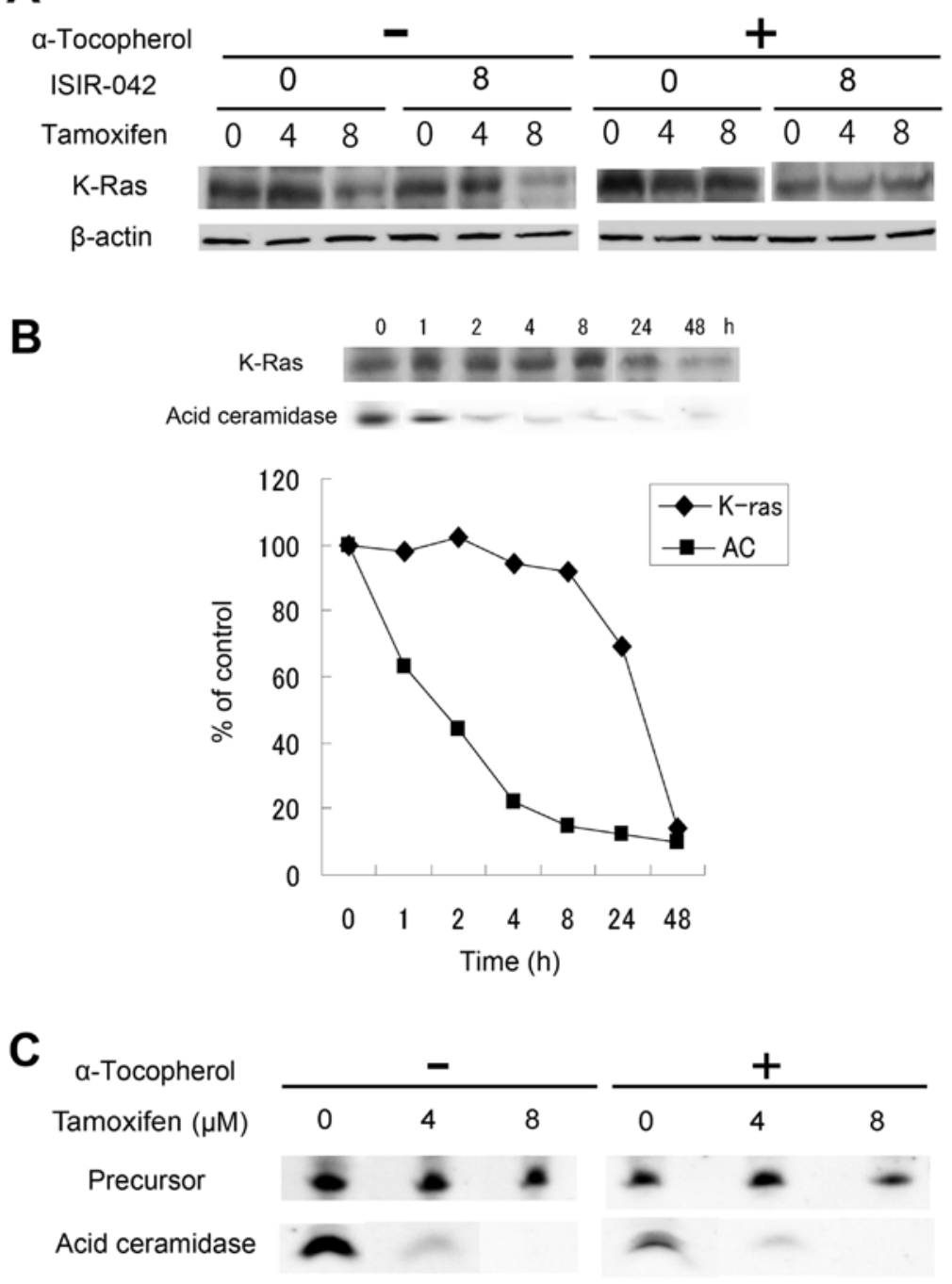

Figure 3. Effect of tamoxifen on the degradation of K-Ras and acid ceramidase (AC). (A) MIAPaCa-2 cells were treated with tamoxifen in the presence or absence of $200 \mu \mathrm{M} \alpha$-tocopherol for $48 \mathrm{~h}$. (B) Time-course of the degradation of K-Ras and AC in MIAPaCa- 2 cells treated with $8 \mu \mathrm{M}$ tamoxifen. (C) Effect of $\alpha$-tocopherol on the degradation of $\mathrm{AC}$ induced by tamoxifen. Cells were treated with various concentrations of tamoxifen in the presence or absence of $\alpha$-tocopherol for $48 \mathrm{~h}$.

that K-ras degradation is closely associated with growth inhibition in tamoxifen-treated cells. However, no significant K-Ras degradation was observed until $8 \mathrm{~h}$ after treatment with tamoxifen, suggesting that K-Ras degradation is not the cause of growth inhibition, but rather the result of growth inhibition, in tamoxifen-treated cells (Fig. 3B).

Acid ceramidase (AC) is a central player in ceramide metabolism. Since AC regulates the levels of pro-apoptotic ceramide and mitogenic sphingosine-1-phosphate, it is considered to be an apt target in cancer therapy. A recent study indicated that tamoxifen induces the downregulation of $\mathrm{AC}$ protein in cancer cells including pancreatic cancer cells (13). Therefore, we examined the effect of tamoxifen on $\mathrm{AC}$ protein in MIAPaCa-2 cells (Fig. 3B and C). A significant downregulation of $\mathrm{AC}$ protein was observed within $1 \mathrm{~h}$, suggesting that this is an early event in the action of tamoxifen (Fig. 3B). However, $\alpha$-tocopherol did not affect the tamoxifen-induced degradation of AC protein (Fig. 3C), suggesting that the downregulation of $\mathrm{AC}$ protein was not associated with the growth inhibition induced by tamoxifen. Tamoxifen induces apoptosis in cancer cells via nitric oxide (NO)-dependent pathways (15). Exogenously added NO potentiated tamoxifen-induced apoptosis and the inhibition of NO synthase (NOS) activity prevented the apoptosis induced by tamoxifen (15). Tamoxifen also induces oxidative stress and the release of cytochrome $\mathrm{c}$ from mitochondria by mechanisms that resemble those of mitochondrial NOS-derived peroxynitrite formation (16).

$\alpha$-tocopherol is a membrane stabilizer and a lipophilic antioxidant (17). Therefore, we examined the effects of tamoxifen on lipid peroxidation and cytochrome c release in the presence or absence of $\alpha$-tocopherol. Stimulation of mitochondrial NOS increases mitochondrial peroxynitrite, which releases cytochrome c from the mitochondria (18). The stimulation of mitochondrial NOS also increases lipid peroxidation (LPO), which is a widely used biomarker of peroxynitrite $(18,19)$. Fig. 4A shows that tamoxifen increased LPO within $4 \mathrm{~h}$ of treatment in MIAPaCa- 2 cells and that the effects of tamoxifen were prevented when samples were co-treated with $\alpha$-tocopherol. The increase in LPO in mitochondria induced by tamoxifen was more evident than that in 
A
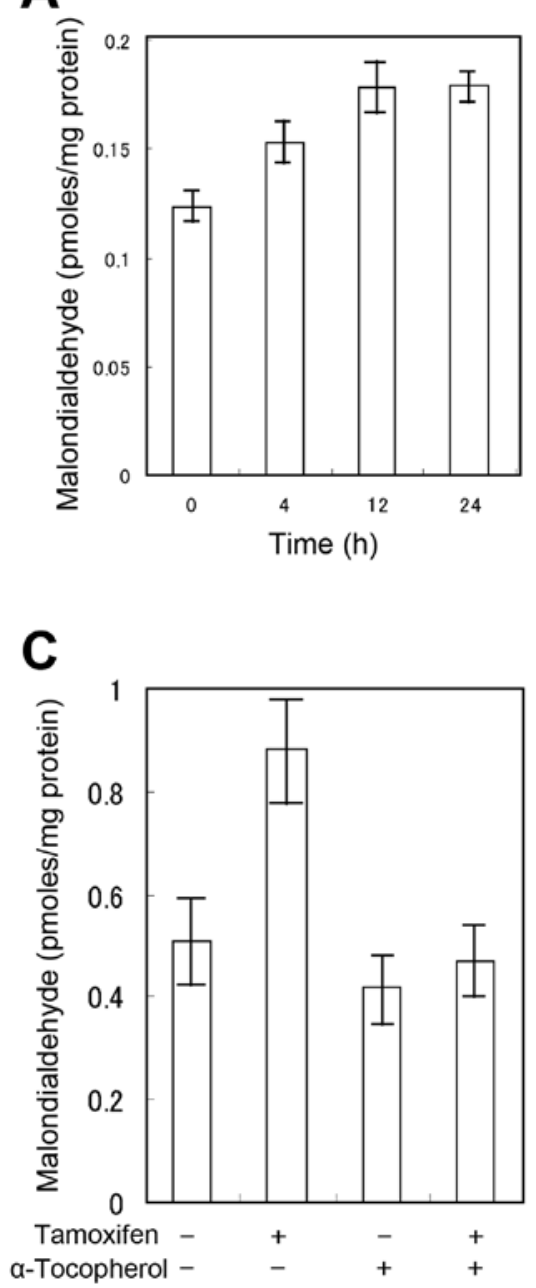

B

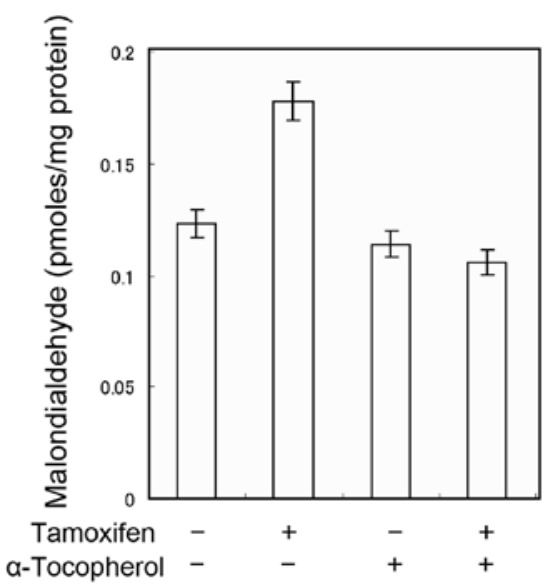

D

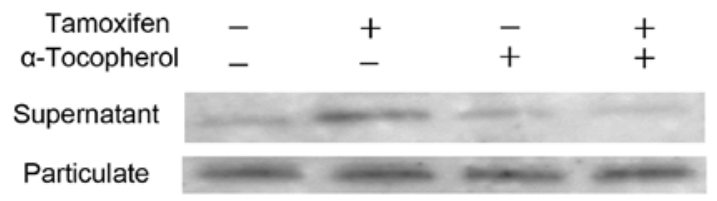

Figure 4. Tamoxifen induces lipid peroxidation (LPO) and cytochrome c release. MIAPaCa-2 cells were treated with $4 \mu \mathrm{M}$ tamoxifen, and LPO was determined by measuring thiobarbituric acid-reactive substances (TBARS). (A and B) LPO in whole cell extracts, (C) LPO in mitochondrial fractions. (D) Cytochrome c release. (B-D) Cells were treated with or without $200 \mu \mathrm{M} \alpha$-tocopherol for $24 \mathrm{~h}$. The values are means of four separate experiments \pm SD.

whole cells (Fig. 4B and C). Furthermore, tamoxifen released cytochrome $\mathrm{c}$ from the particulate fraction which included mitochondria in MIAPaCa-2 cells (Fig. 4D).

Effect of ISIR-042 on colony formation by MIAPaCa-2 cells and the expression of stemness-related genes. Pancreatic cancer stem cells express the cell surface markers CD24 and CD44 (20,21). Gemcitabine showed an increased percentage of CD $24^{+} \mathrm{CD} 44^{+}$cells in MIAPaCa- 2 cells, whereas ISIR-042 significantly reduced the percentage of $\mathrm{CD} 24^{+} \mathrm{CD} 44^{+}$cells, suggesting that ISIR-042 preferentially inhibits stem/progenitor cells in pancreatic cancer cells (5). Therefore, we examined the effects of ISIR-042 on colony formation by MIAPaCa- 2 cells in semi-solid culture and the expression of stemness-related genes. ISIR-042 effectively inhibited colony formation by MIAPaCa- 2 cells in semi-solid culture, and the formation of large colonies was inhibited more evidently than that of small colonies (Fig. 5A). Colony formation in semi-solid culture was more efficiently inhibited by ISIR-042 than cell proliferation in liquid culture, whereas the inhibition of colony formation by tamoxifen or 5FU was similar to the inhibition of cell proliferation (Fig. 5B).
The growth of pancreatic cancer cell lines was effectively inhibited by ISIR-042, whereas only weak growth-inhibition was seen in normal endothelial HUVEC cells (5). Cotylenin A, the parent compound of ISIR-042, preferentially induced apoptosis in human cancer cells, while sparing normal cells (22). We examined the effects of ISIR-042 on colony formation by normal mouse bone marrow cells in the presence or absence of tamoxifen. Although ISIR-042 alone hardly affected colony formation by bone marrow cells, the combination of ISIR-042 and $4 \mu \mathrm{M}$ tamoxifen only weakly inhibited colony formation by normal bone marrow cells (Fig. 5C). These results suggest that pancreatic cancer cells were more sensitive to combined treatment with ISIR-042 and tamoxifen than normal bone marrow cells.

Since the expression of pluripotency-associated transcription factors has been implicated in the maintenance of cancer stem cells, we evaluated mRNA expression of Sox-2, Oct3/4 and Nanog (Fig. 5D). The expression of Sox-2 and Nanog mRNAs was significantly suppressed by treatment with ISIR042. This reduction was observed within 4 days. ISIR-042 did not significantly affect the expression of Oct3/4 mRNA under these conditions. Tamoxifen also reduced the expression level 
A
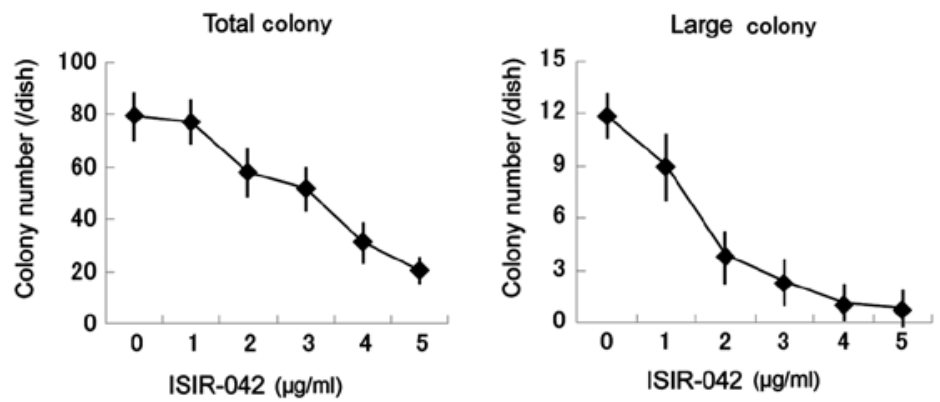

B
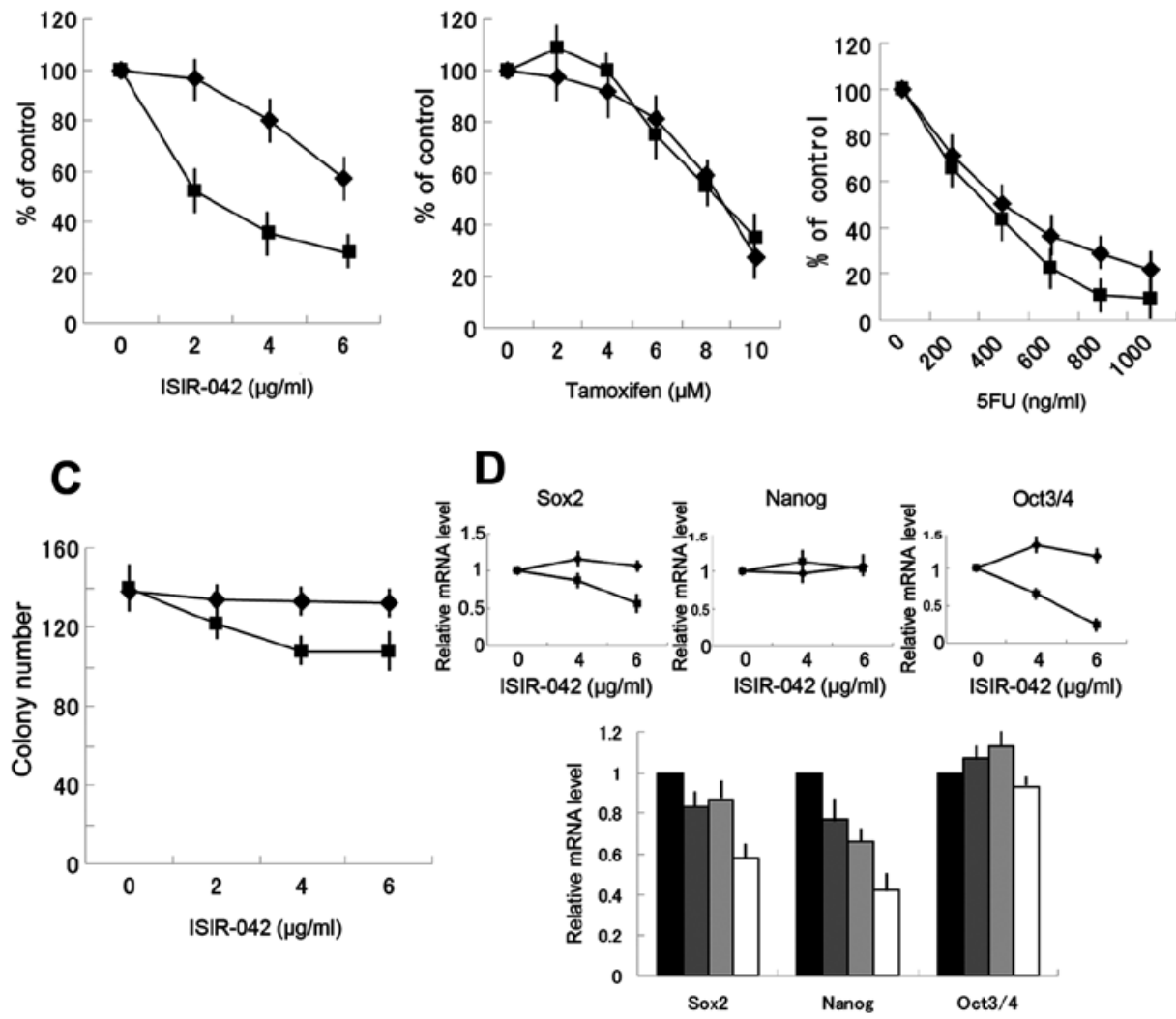

Figure 5. (A) Effect of ISIR-042 on colony formation by MAIPaCa-2 cells in semi-solid culture. Large colony; $>0.7$ mm in diameter. The values are means of nine separate experiments \pm SD. (B) Effects of ISIR-042, tamoxifen or 5FU on cell proliferation in liquid culture ( $\bullet$ ) and colony formation by MIAPaCa- 2 cells in semi-solid culture ( $\mathbf{\square})$. The values are means of six separate experiments \pm SD. (C) Effect of ISIR-042 on colony formation by normal mouse bone marrow cells in the presence $(\mathbf{\square})$ or absence $(\bullet)$ of $4 \mu \mathrm{M}$ tamoxifen. The values are means of six separate experiments \pm SD. (D) Effect of ISIR-042 on gene expression of stemness-related transcription factors. Cells were treated with ISIR-042 for 2 ( $\bullet$ ) or 4 (घ) days. Combined effects of ISIR-042 and tamoxifen on gene expression of stemness-related transcription factors. Cells were treated with (light grey, $\square$ ) or without ( $\mathbf{\square}$, grey) $4 \mu \mathrm{g} / \mathrm{ml}$ ISIR-042 in the presence (grey, $\square$ ) or absence ( $\mathbf{n}$, light grey) of $4 \mu \mathrm{M}$ tamoxifen for 4 days. The values are means of six separate experiments \pm SD.

of Nanog mRNA, although the effects were less than those of ISIR-042 (Fig. 5D).

Combined effects of tamoxifen, ISIR-042 and anticancer drugs on growth inhibition and the appearance of drugresistant cells. The combined effects of ISIR-042, tamoxifen and anticancer drugs were examined. A triple combination (ISIR-042, tamoxifen and 5FU) cooperatively inhibited the growth of MIAPaCa-2 cells (Fig. 6A). 5FU at 1,000 ng/ml did not completely inhibit cell viability, and the combinations of $5 \mathrm{FU}$ and $6 \mu \mathrm{g} / \mathrm{ml}$ of ISIR-042 and the combination of $5 \mathrm{FU}$ and $4 \mu \mathrm{M}$ tamoxifen did not produce $100 \%$ killing either. However, treatment with the triple combination led to no viable cells in the culture (as measured by MTT assay). Similar results were obtained when cells were treated with gemcitabine. Next, we examined the growth-inhibitory effects of triple combinations in long-term culture. The growth of Panc-1 cells was markedly reduced for 12 days by treatment with $500 \mathrm{ng} / \mathrm{ml}$ of $5 \mathrm{FU}$ alone. However, after $\sim 16$ days, growing cells reappeared and the proliferation rate of the treated cells became similar to that of untreated cells (Fig. 6B). Drug-resistant cells were not observed when cells were cultured for 28 days with $500 \mathrm{ng} / \mathrm{ml}$ 5FU, $3 \mu \mathrm{M}$ tamoxifen and $3 \mu \mathrm{g} / \mathrm{ml}$ ISIR-042, and dual combinations (5FU+tamoxifen or 5FU+ISIR042) were less effective. 
A

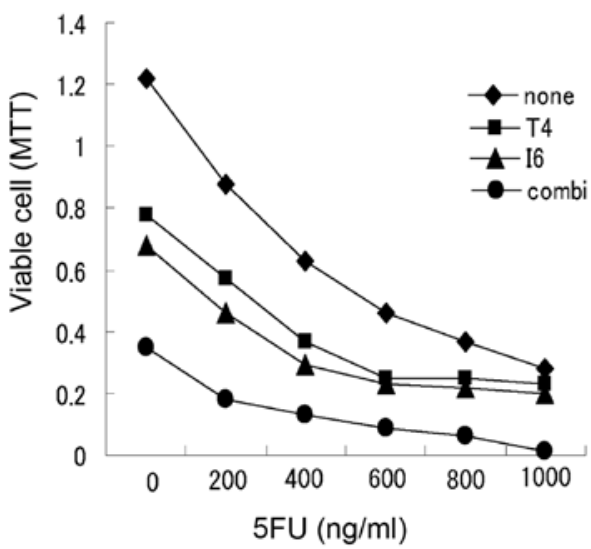

B

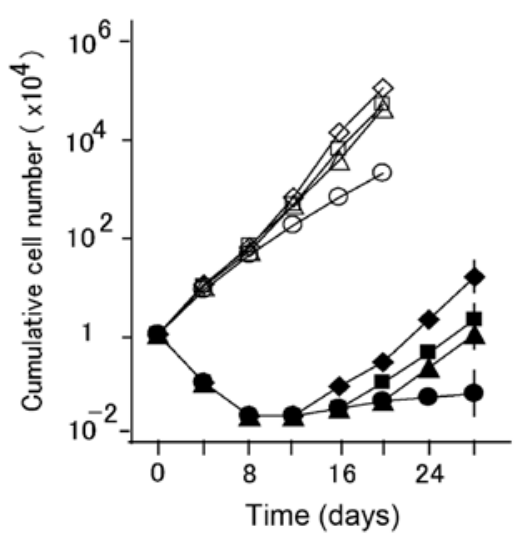

C

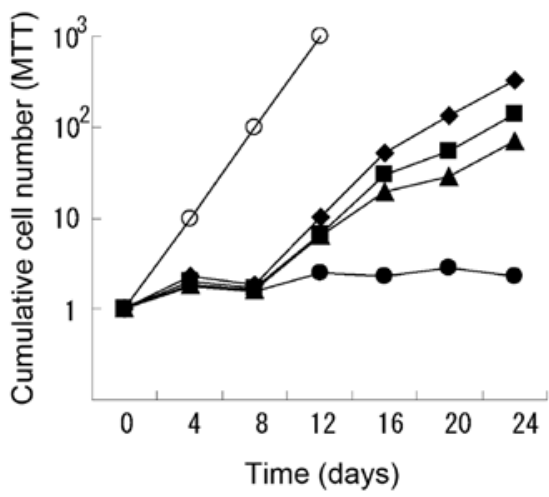

D

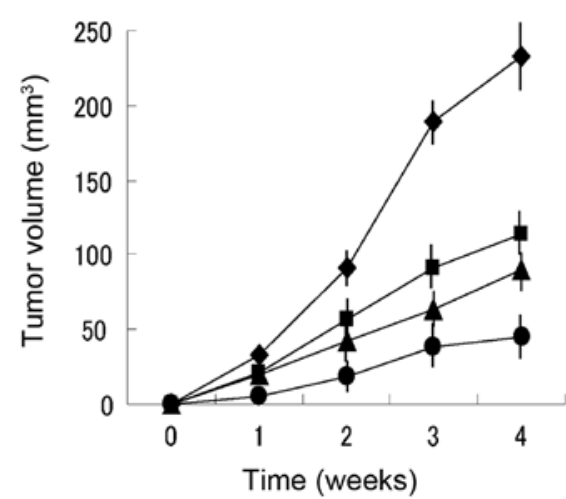

E

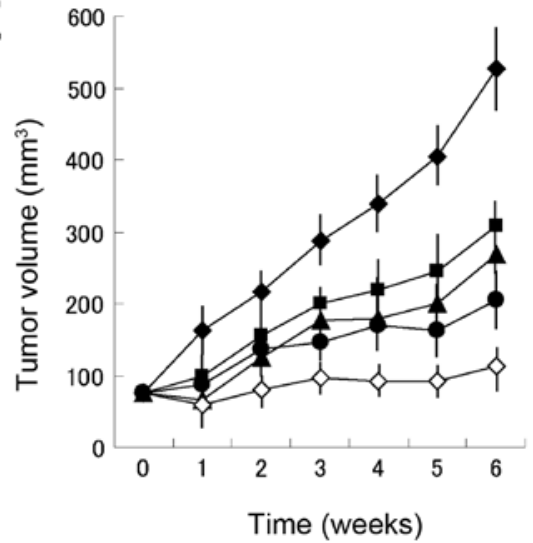

Figure 6. Combined effects of tamoxifen, ISIR-042 and anticancer drugs on the growth of pancreatic cancer cells. (A) Combined effects of tamoxifen, ISIR-042 and 5FU on the growth of MIAPaCa-2 cells. Cells were cultured with various concentrations of 5FU in the absence ( $\bullet)$ or presence of $4 \mu \mathrm{M}$ tamoxifen $(\bullet), 6 \mu \mathrm{g} / \mathrm{ml}$ of ISIR-042 (४), or $4 \mu \mathrm{M}$ tamoxifen $+6 \mu \mathrm{g} / \mathrm{ml}$ of ISIR-042 $(\bullet)$ for 5 days. (B) Combined treatment with tamoxifen and ISIR-042 prevents the appearance of 5FU-resitant cells. Panc-1 cells were cultured without $(\diamond, \bullet)$ or with $3 \mu \mathrm{M}$ tamoxifen $(\square, \mathbf{\square}), 3 \mu \mathrm{g} / \mathrm{ml}$ of ISIR-042 $(\Delta, \mathbf{\Lambda})$, or $3 \mu \mathrm{M}$ tamoxifen $+3 \mu \mathrm{g} / \mathrm{ml}$ of ISIR-042 (०, $\bullet$ ). Cells in closed symbols were treated with $500 \mathrm{ng} / \mathrm{ml}$ of $5 \mathrm{FU}$. The culture medium was replaced by fresh medium with drugs every 4 days and the cell density was kept so as to maintain the growth phase. (C) Combined treatment with tamoxifen and ISIR-042 prevents the appearance of gemcitabine-resistant cells. Panc-1 cells were cultured without $(\bullet)$ or with $4 \mu \mathrm{M}$ tamoxifen $(\boldsymbol{\bullet}), 4 \mu \mathrm{g} / \mathrm{ml}$ of ISIR-042 ( $\mathbf{\Delta})$, or $4 \mu \mathrm{M}$ tamoxifen $+4 \mu \mathrm{g} / \mathrm{ml}$ of ISIR-042 (•). Cells in closed symbols were treated with $1 \mathrm{nM}$ of gemcitabine. $\circ$, Untreated control. The culture medium was replaced by fresh medium with drugs every 4 days and the cell density was kept so as to maintain cells in the growth phase. (D) Effects of tamoxifen and ISIR-042 on the growth of Panc-1 cells as xenografts. Treatment was initiated 3 days after tumor inoculation. Mice were given daily i.p. injections of $3 \mathrm{mg} / \mathrm{kg}$ ISIR-042 in $0.2 \mathrm{ml} \mathrm{PBS} \mathrm{( \mathbf { \Lambda } , \bullet )}$ and/or $2 \mathrm{mg} / \mathrm{kg}$ tamoxifen in $0.1 \mathrm{ml}$ corn oil $(\mathbf{\bullet}, \bullet)$. $\bullet$, Untreated mice. (E) Effects of tamoxifen, ISIR-042 and 5FU on the growth of Panc-1 cells as xenografts. Treatment was initiated when tumors in each group achieved an average volume of $80 \mathrm{~mm}^{3}$. Treatment with $5 \mathrm{mg} / \mathrm{kg} 5 \mathrm{FU}$ was performed three times per week $(\bullet, \Delta, \bullet, \diamond)$. Mice were given daily i.p. injections of $3 \mathrm{mg} / \mathrm{kg}$ ISIR-042 in $0.2 \mathrm{ml}$ PBS $(\bullet, \diamond)$ and $/$ or $2 \mathrm{mg} / \mathrm{kg}$ tamoxifen in $0.1 \mathrm{ml}$ corn oil $(\mathbf{\Delta}, \diamond)$. $\bullet$ Untreated mice. The values are means $( \pm \mathrm{SD})$ of 10 mice.

Similar results were obtained in the long-term culture of cells treated with gemcitabine (Fig. 6C). These results suggest that the triple combination therapy is effective for overcoming the emergence of drug-resistant cells in pancreatic cancers.
Effects of tamoxifen, ISIR-042 and 5FU on the in vivo growth of pancreatic cancer cells as xenografts. The in vitro studies described above suggested that treatment with the triple combination should be more effective therapeutically than treatment 
with 5FU alone. Treatments were started at day 2 after the inoculation of Panc-1 cells. We administered both tamoxifen and ISIR-042 daily by intraperitoneal injection. The combined treatment had no appreciable adverse effects in mice, including weight loss. Although neither ISIR-042 nor tamoxifen alone retarded tumor growth, combined treatment was more effective (Fig. 6D). Next, we examined the antitumor effects of the combination of 5FU with tamoxifen and ISIR-042. Treatment was initiated when tumors in each group achieved an average volume of $80 \mathrm{~mm}^{3}$. Mice were treated with $5 \mathrm{mg} / \mathrm{kg} 5 \mathrm{FU}$ three times per week.

While treatment with 5FU alone induced a slight loss of body weight (6-8\% loss), treatment with the triple combination did not induce a further reduction of body weight. Although 5FU alone retarded tumor growth, combined treatment with 5FU, tamoxifen and ISIR-042 was more effective (Fig. 6E). Statistical analysis (F-test) revealed that the difference was significant $(\mathrm{P}<0.05$ at 6 weeks). There were no significant differences between treatment with 5FU and combination treatment with 5FU+tamoxifen or 5FU+ISIR042. These results indicate that the combination of tamoxifen and ISIR-042 is effective therapeutically, consistent with the in vitro findings.

\section{Discussion}

Tamoxifen induces apoptosis in both ER-positive and ER-negative cells (6). Treatment of human pancreatic cancer cells with 2-8 $\mu \mathrm{M}$ tamoxifen, concentrations which are 6-25 times greater than the therapeutic concentration (the average therapeutic blood concentration in patients with breast carcinoma was $0.3 \mu \mathrm{M}$ ) inhibited cell growth. Treatment with $\alpha$-tocopherol prevented the growth-inhibition induced by tamoxifen (Fig. 3A). Submicromolar concentrations of tamoxifen released cytochrome $\mathrm{c}$ from isolated mitochondria and increased LPO in isolated mitochondria (16). The release of cytochrome $\mathrm{c}$ and increased LPO in tamoxifen-treated cells was inhibited by $\alpha$-tocopherol (Fig. 5). Since the action of tamoxifen on cancer cells is counteracted by the potent membrane stabilizer $\alpha$-tocopherol, the main mechanism of action of tamoxifen is considered to be membrane damage via LPO. These results suggest that tamoxifen is an anticancer drug that induces oxidative stress and apoptosis via a mitochondria-dependent pathway.

The novel fusicoccin derivative ISIR-042 was synthesized from fusicoccin A as an antitumor drug (5). A receptor of fusicoccin has been reported to be a member of a family of 14-3-3 proteins that are found commonly in a huge array of signaling and regulatory pathways (23). The $14-3-3$ proteins bind to discrete phosphoserin-containing motifs that are present in many signaling molecules. The 14-3-3 proteins are associated with dynamic nucleocytoplasmic shuttling. Phosphorylationdependent binding of 14-3-3 proteins to telomerase promotes its nuclear localization (24). However, the reverse has more often been the case, in that many nuclear proteins can become phosphorylated, bind to 14-3-3 proteins and accumulate in the cytoplasm. The 14-3-3 proteins negatively regulate histone deacetylase 4 (25) or the cyclin-dependent kinase inhibitor p27 (26) by preventing its nuclear localization. Fusicoccins bind to inhibitory 14-3-3 interaction sites of c-RAF, pSer233 and pSer259, but not to the activating interaction site, pSer621 (27). The modification by fusicoccins may lead to the upregulation of apoptosis-regulating genes and the downregulation of cell cycle- and stemness-regulating genes. Our previous findings indicate that the pancreatic cancer stem cell marker CD $24^{+} \mathrm{CD} 44^{+}$is significantly reduced by ISIR-042 in a MIAPaCa-2 cell population (5).

In the present study, ISIR-042 preferentially inhibited the population that formed large colonies, which may be cells with high self-renewal activity. Treatment with ISIR-042 effectively downregulated the stemness-associated transcription factors Sox-2 and Nanog. Expression of these genes is important for the development of tumors, as shown in in vivo experiments. Knockdown of Nanog expression inhibits the stemness of pancreatic cancer cells (28). Sox-2 was highly expressed in the clonogenic compartment of pancreatic cancer cells and targeting of Sox-2 could be a worthy strategy for pancreatic cancer therapy (29). Pancreatic carcinoma remains incurable for the vast majority of patients, suggesting that pancreatic cancer stem cells are relatively resistant to chemotherapy. ISIR-042 effectively inhibits clonogenic growth and the expression of stemnessrelated genes in pancreatic cancer cells. ISIR-042 effectively sensitizes pancreatic cancer cells to $5 \mathrm{FU}$ or gemcitabine (5). These results suggest that ISIR-042 is a useful drug for cancer stem cell-targeted therapy against pancreatic carcinoma.

After estrogen receptors were detected in human pancreatic carcinomas (30-32), tamoxifen was evaluated in several trials. These studies reported an increased survival time in patients with resected and non-resected pancreatic cancer after treatment with tamoxifen (33-35). Furthermore, phase II trials of tamoxifen with 5FU or gemcitabine have been performed in pancreatic cancer $(36,37)$. These data suggest that tamoxifen has some positive effects in the treatment of pancreatic cancer, although the effect is modest. The present results suggest that the addition of ISIR-042 to tamoxifen potentiates its antiproliferative action, which provides a synergistic effect. Toxicity studies with mice and rats have suggested that ISIR042 is well-tolerated (5). Furthermore, combined treatment with tamoxifen and ISIR-042 did not further affect the toxicity of 5FU, as judged by body weight loss. Supplementation of 5FU- or gemcitabine-based chemotherapy against pancreatic cancers with tamoxifen and ISIR-042 may provide a stronger beneficial effect.

\section{Acknowledgements}

This work was supported in part by the SUIGAN project, Shimane University, Japan.

\section{References}

1. Evance DB, Abbruzzes JL and Rich TA: Cancer of the pancreas. In: Cancer, Principles and Practice of Oncology. DeVita VT Jr, Hellman S and Rosenberg SA (eds). J.B. Lippincott Co., Philadelphia, pp1054-1087, 1997.

2. Siegel R, Naishadham D and Jemal A: Cancer statistics, 2012. CA Cancer J Clin 62: 10-29, 2012.

3. Conroy T, Desseigne F, Ychou M, Bouché O, Guimbaud R, Bécouarn Y, Adenis A, Raoul JL, Gourgou-Bourgade S, de la Fouchardière $\mathrm{C}$, et al; Groupe Tumeurs Digestives of Unicancer; PRODIGE Intergroup: FOLFIRINOX versus gemcitabine for metastatic pancreatic cancer. N Engl J Med 364: 1817-1825, 2011. 
4. Bozic I, Reiter JG, Allen B, Antal T, Chatterjee K, Shah P, Moon YS, Yaqubie A, Kelly N, Le DT, et al: Evolutionary dynamics of cancer in response to targeted combination therapy. eLife 2: e00747, 2013.

5. Kawakami K, Hattori M, Inoue T, Maruyama Y, Ohkanda J, Kato N, Tongu M, Yamada T, Akimoto M, Takenaga K, et al: A novel fusicoccin derivative preferentially targets hypoxic tumor cells and inhibits tumor growth in xenografts. Anticancer Agents Med Chem 12: 791-800, 2012.

6. Mandlekar S and Kong ANT: Mechanisms of tamoxifen-induced apoptosis. Apoptosis 6: 469-477, 2001.

7. Avgeropoulos NG and Batchelor TT: New treatment strategies for malignant gliomas. Oncologist 4: 209-224, 1999.

8. Chou TC and Talalay P: Quantitative analysis of dose-effect relationships: The combined effects of multiple drugs or enzyme inhibitors. Adv Enzyme Regul 22: 27-55, 1984.

9. Buege JA and Aust SD: Microsomal lipid peroxidation. Methods Enzymol 52: 302-310, 1978.

10. Tian Y, Ding W, Wang Y, Ji T, Sun S, Mo Q, Chen P, Fang Y, Liu J, Wang B, et al: Ubiquitin B in cervical cancer: Critical for the maintenance of cancer stem-like cell characters. PLoS One 8: e84457, 2013.

11. Hattori M, Kawakami K, Akimoto M, Takenaga K, Suzumiya J and Honma Y: Antitumor effect of Japanese apricot extract (MK615) on human cancer cells in vitro and in vivo through a reactive oxygen species-dependent mechanism. Tumori 99: 239-248, 2013

12. Kohli L, Kaza N, Coric T, Byer SJ, Brossier NM, Klocke BJ, Bjornsti MA, Carroll SL and Roth KA: 4-Hydroxytamoxifen induces autophagic death through K-Ras degradation. Cancer Res 73: 4395-4405, 2013.

13. Morad SAF, Levin JC, Tan S-F, Fox TE, Feith DJ and Cabot MC: Novel off-target effect of tamoxifen - inhibition of acid ceramidase activity in cancer cells. Biochim Biophys Acta 1831: 1657-1664, 2013.

14. Hezel AF, Kimmelman AC, Stanger BZ, Bardeesy N and Depinho RA: Genetics and biology of pancreatic ductal adenocarcinoma. Genes Dev 20: 1218-1249, 2006.

15. Maccarrone M, Fantini C, Ranalli M, Melino G and Agrò AF Activation of nitric oxide synthase is involved in tamoxifeninduced apoptosis of human erythroleukemia K562 cells. FEBS Lett 434: 421-424, 1998.

16. Nazarewicz RR, Zenebe WJ, Parihar A, Larson SK, Alidema E, Choi J and Ghafourifar P: Tamoxifen induces oxidative stress and mitochondrial apoptosis via stimulating mitochondrial nitric oxide synthase. Cancer Res 67: 1282-1290, 2007.

17. Atkinson J, Harroun T, Wassall SR, Stillwell W and Katsaras J: The location and behavior of alpha-tocopherol in membranes. Mol Nutr Food Res 54: 641-651, 2010.

18. Ghafourifar P, Schenk U, Klein SD and Richter C: Mitochondrial nitric-oxide synthase stimulation causes cytochrome c release from isolated mitochondria. Evidence for intramitochondrial peroxynitrite formation. J Biol Chem 274: 31185-31188, 1999.

19. Radi R, Beckman JS, Bush KM and Freeman BA: Peroxynitriteinduced membrane lipid peroxidation: The cytotoxic potential of superoxide and nitric oxide. Arch Biochem Biophys 288: 481-487, 1991

20. Li C, Heidt DG, Dalerba P, Burant CF, Zhang L, Adsay V, Wicha M, Clarke MF and Simeone DM: Identification of pancreatic cancer stem cells. Cancer Res 67: 1030-1037, 2007.

21. Lee CJ, Li C and Simeone DM: Human pancreatic cancer stem cells: Implications for how we treat pancreatic cancer. Transl Oncol 1: 14-18, 2008.
22. Honma Y, Ishii Y, Yamamoto-Yamaguchi Y, Sassa $T$ and Asahi K: Cotylenin A, a differentiation-inducing agent, and IFN-alpha cooperatively induce apoptosis and have an antitumor effect on human non-small cell lung carcinoma cells in nude mice. Cancer Res 63: 3659-3666, 2003.

23. Oecking C, Eckerskorn $\mathrm{C}$ and Weiler EW: The fusicoccin receptor of plants is a member of the 14-3-3 superfamily of eukaryotic regulatory proteins. FEBS Lett 352: 163-166, 1994.

24. Seimiya H, Sawada H, Muramatsu Y, Shimizu M, Ohko K, Yamane $\mathrm{K}$ and Tsuruo T: Involvement of 14-3-3 proteins in nuclear localization of telomerase. EMBO J 19: 2652-2661, 2000.

25. Rittinger K, Budman J, Xu J, Volinia S, Cantley LC, Smerdon SJ, Gamblin SJ and Yaffe MB: Structural analysis of 14-3-3 phosphopeptide complexes identifies a dual role for the nuclear export signal of 14-3-3 in ligand binding. Mol Cell 4: 153-166, 1999.

26. Fujita N, Saito S and Tsuruo T: Phosphorylation of p27Kip1 at threonine 198 by p90 ribosomal protein S6 kinase promotes its binding to 14-3-3 and cytoplasmic localization. J Biol Chem 278: 49254-49260, 2003

27. Molzan M, Kasper S, Röglin L, Skwarczynska M, Sassa T, Inoue T, Breitenbuecher F, Ohkanda J, Kato N, Schuler M, et al: Stabilization of physical RAF/14-3-3 interaction by cotylenin A as treatment strategy for RAS mutant cancers. ACS Chem Biol 8: 1869-1875, 2013

28. Lu Y, Zhu H, Shan H, Lu J, Chang X, Li X, Lu J, Fan X, Zhu S, Wang Y, et al: Knockdown of Oct4 and Nanog expression inhibits the stemness of pancreatic cancer cells. Cancer Lett 340: 113-123, 2013.

29. Herreros-Villanueva M, Bujanda L, Billadeau DD and Zhang J-S: Embryonic stem cell factors and pancreatic cancer. World J Gastroenterol 20: 2247-2254, 2014

30. Greenway B, Iqbal MJ, Johnson PJ and Williams R: Oestrogen receptor proteins in malignant and fetal pancreas. Br Med J (Clin Res Ed) 283: 751-753, 1981

31. Benz C, Hollander C and Miller B: Endocrine-responsive pancreatic carcinoma: Steroid binding and cytotoxicity studies in human tumor cell lines. Cancer Res 46: 2276-2281, 1986.

32. Singh S, Baker PR, Poulsom R, Wright NA, Sheppard MC, Langman MJ and Neoptolemos JP: Expression of oestrogen receptor and oestrogen-inducible genes in pancreatic cancer. Br J Surg 84: 1085-1089, 1997.

33. Mikulski S: Tamoxifen in patients with advanced pancreatic adenocarcinoma. Oncol Rep 3: 17-19, 1996.

34. Horimi T, Takasaki M, Toki A, Nishimura W and Morita S: The beneficial effect of tamoxifen therapy in patients with resected adenocarcinoma of the pancreas. Hepatogastroenterology 43: 1225-1229, 1996.

35. Lamy R, Conroy T, Brunaud L and Bresler L: Tamoxifen for metastatic pancreatic adenocarcinoma: A complete response. Gastroenterol Clin Biol 25: 912-913, 2001 (In French).

36. Eckel F, Lersch C, Lippl F, Assmann G and Schulte-Frohlinde E: Phase II trial of cyclophosphamide, leucovorin, 5-fluorouracil 24-hour infusion and tamoxifen in pancreatic cancer. J Exp Clin Cancer Res 19: 295-300, 2000.

37. Tomao S, Romiti A, Massidda B, Ionta MT, Farris A, Zullo A, Brescia A, Santuari L and Frati L: A phase II study of gemcitabine and tamoxifen in advanced pancreatic cancer. Anticancer Res 22: 2361-2364, 2002 